\title{
Supernovae and the Galactic Ecosystem
}

\author{
Q. Daniel Wang \\ Astronomy Department, \\ 619-E, LGRT, University of Massachusetts, 710 N. Pleasant St., Amherst, MA 01003, USA \\ email: wqd@astro.umass.edu
}

\begin{abstract}
Supernovae are the dominant source of stellar feedback, which plays an important role in regulating galaxy formation and evolution. While this feedback process is still quite uncertain, it is probably not due to individual supernova remnants as commonly observed. Most supernovae likely take place in low-density, hot gaseous environments, such as superbubbles and galactic bulges, and typically produce no long-lasting bright remnants. I review recent observational and theoretical work on the impact of such supernovae on galaxy ecosystems, particularly on hot gas in superbubbles and galactic spheroids.
\end{abstract}

Keywords. ISM: general, galaxies: ISM, X-rays: galaxies, (ISM:) supernova remnants

\section{Introduction}

Supernovae (SNe) are a major source of the mechanical energy input in the interstellar medium (ISM). On average, an SN releases about $10^{51}$ ergs energy, driving a blastwave into the ambient medium. How far this blastwave goes and how fast the energy is dissipated depend sensitively on the density and temperature of the medium. Ironically, commonly-known and well-studied supernova remnants (SNRs), though looking spectacular, are atypical products of $\mathrm{SNe}$, and typically occur in relatively dense media. Most $\mathrm{SNe}$ are expected to explode in low-density hot environments, e.g., inside superbubbles (SBs) and/or simply in the inter-cloud hot ISM (for core-collapsed SNe) and in the Galactic halo and bulge (for Type Ia SNe). The resultant remnants are typically too faint to be well observed individually. But collectively such "missing" SNRs are probably more important than those in dense environments, in terms of both heating and shaping the global ISM. In the following, I will first briefly review the behavior of SN blastwaves in hot gas and will then focus on discussing how discrete SN events affect hot gas properties in the two types of environments, SBs and galactic spheroids.

\section{Supernova blastwaves in hot gas}

As shown by Tang \& Wang (2005), the evolution of an SNR in a low-density hot medium has several distinct properties: 1) The blastwave always moves at a speed greater than, or comparable to, the sound wave and can thus reach a much larger radius than that predicted by the Sedov solution; 2) The swept-up thermal energy is important, affecting the evolution of both the blastwave and the interior structure; 3) Because the Mach number of the blastwave is typically small, its heating is subtle and over a large volume; 4) The blastwave can hardly dissipate until it meets cool gas. These properties of SNRs in a low-density hot medium make them an important ingredient in regulating large-scale environments in galaxies.

To study the collective effect of SNe on the ISM, we need to conduct simulations, which needs to cover a large dynamic range from the evolution of individual SNRs to a possible galaxy-wide outflow, for example. It would be computationally very expensive, if even 
possible, to simulate the evolution of each SNR on sub-parsec scales in such a simulation. The SNR evolution in general cannot be described by the self-similar Sedov Taylor solution, which neglects the SN ejecta and assumes a cool ambient medium (hence with no energy content). In fact, the evolution depends on both the density and temperature of the ambient medium (Tang \& Wang 2005). Tang \& Wang (2009) have further shown that one can adaptively embed individual structured SNR seeds into the 3D simulation grid on such scales that their thermal and chemical evolution are adequately represented by detailed 1D simulations. The SNR seed embedding, worked with an adaptive mesh refinement scheme, can effectively extend such a 3D simulation to include the subgrid evolution of SNRs. In fact, using a scaling law, which is applicable to SNRs evolving in hot gas, individual seeds can be adaptively generated from a library of templates. Each consists of the radial profiles of density, temperature and velocity when the SNR has a certain shock front radius or age. These templates can be obtained from a single 1-D simulation of an SNR evolving in a uniform ambient medium of certain density and temperature. With this scheme, 3D hydrodynamic simulations of the supernova-dominated ISM can be readily conducted.

\section{Superbubbles}

Core-collapsed SNe represent the end of massive stars, the bulk (if not all) of which form in $\mathrm{OB}$ associations. The energy release from such an association, highly correlated in space and time, has a great impact on the surrounding medium. Initially, the energy release from the association is primarily in the form of intense ionizing radiation from very massive stars, which tends to homogenize the surrounding medium and to make it puff up. After several million years, fast stellar winds start to play a major role in heating and shaping the medium, creating a low-density hot bubble (e.g., Weaver et al. (1977)), before the explosion of the first SN in the association. Later, after about $5 \times 10^{6} \mathrm{yr}$ since the star formation (if more or less coeval), core-collapsed SNe become the dominant source of the mechanical energy input into the already hot surroundings (e.g., Monaco 2004). This combination of the concerted feedbacks, lasting for $\sim 5 \times 10^{7} \mathrm{yr}$ - the lifetime of an $8 M_{\odot}$ star - leads to the formation of a so-called superbubble of low-density hot gas enclosed by a supershell of swept-up cool gas (e.g., Mac Low \& McCray 1988). The expansion of such a superbubble is expected to be substantially faster than typical OB association internal velocities of a few kilometers per second. Therefore, a majority of core-collapsed SNe ( 90\%) should occur inside their parent superbubbles (e.g., Higdon et al. 1998; Parizot et al. 2004).

Fig. 1 shows examples of SBs in various evolutionary stages. The 30 Dor nebula is probably the youngest and most energetic one, while 30 Dor $\mathrm{C}$ is the oldest and least powerful. The diffuse X-ray-emitting plasma, heated by fast stellar winds and possible SNe of massive stars, fills various cavities traced by $\mathrm{H} \alpha$-emitting shells. Such SBs will eventually blow out from galactic disks and vent the hot plasma into galactic halos, even into the intergalactic space.

While the evolution model for SBs, analogous to that for stellar wind bubbles, has been developed for many years (e.g., Weaver et al. 1977; Mac Low \& McCray 1988). Comparisons of this model with observations have revealed two major discrepancies. The observed X-ray luminosities of some SBs seem to be substantially brighter (by a factor of up to $\sim 10$ ) than predicted by the model (Chu \& Mac Low 1990; Wang \& Helfand 1991; Jaskot et al. 2011 and references therein). Various processes have been proposed, including SN metal enrichment, additional mass loading due to the destruction of embedded, dense cloudlets of the ISM that have been overrun by expanding supershells. 
But it appears that the most important process is the blastwave heating of supershells by sporadic SNe, especially off-center ones (Jaskot et al. (2011) and references therein).

The blastwave heating of supershells may also be responsible for another apparent discrepancy between the model prediction and the observations: The energetics of SBs, observationally accounting for both thermal and kinetic energies, is generally much less than expected from the injection from massive stars enclosed (e.g., Yamaguchi et al. (2010)). Or the growth rate of an SB is typically much less than what would be expected from the model (e.g., Oey (2009)). The energy injection from stars is assumed as being continuous, which may be a reasonable approximation for an evolved SB of a large dimension, which the model was intended to applyd to. But for a relatively young and small SB, as typically observed, an SN-induced shockwave or sound wave can propagate through the hot interior, eventually reach the surrounding cool shells, and steepen again into a blastwave, somewhat like an earth quake-induced Tsunami (Tang \& Wang (2005)). This blastwave, or its corresponding reverse-shock, can sometimes be strong enough to produce enhanced X-ray emission, as observed. Furthermore, much of the SN energy may be lost radiatively, probably outside the X-ray band. Indeed, as shown by Cho et al. (2008), based on 1-D simulations of such young SBs, discrete SNe can lead to a loss of $80-90 \%$ of the SN mechanical energy via radiative cooling in supershells, and only about $10 \%$ remains as the thermal energy in the enclosed hot gas, while the kinetic energy is $\lesssim 10 \%$. Of course, such a substantial energy loss can significantly change the growth of SBs, and potentially their chance to blow out from galactic disks. Therefore, it should be interesting to extend such simulations to higher dimensions and to later evolutionary stages of SBs. Interfaces between the hot and cold gases can be enlarged, for example, due to various $3-\mathrm{D}$ instabilities, which can potentially strongly affect the energetics of SBs (e.g., Krause et al. (2013)).

In addition to X-ray emission, SBs may loss energy via other channels. Substantial amounts of dust have been observed in various Galactic wind-blown bubbles (e.g., Everett \& Churchwell 2010). Dust can be a significant sink of heat in hot gas. But the survival time of dust in hot gas is short, due to sputtering. So dust needs to be replenished, possibly by clouds overrun by expanding shells and by dense stellar winds from massive stars such as Carbon-rich Wolf-Rayets and Luminous Blue variables (e.g., Rajagopal et al. 2007, Dong et al. (2012)).

In addition to the radiation loss, $\mathrm{SN}$ energy may also escape from SBs via cosmic rays (CRs). SBs have been proposed to be the acceleration site of Galactic CRs (e.g., Higdon \& Ligenfelter 2005, Fisk \& Gloeckler 2012; Parizot et al. 2004; Bykov 2001; Bykov \&
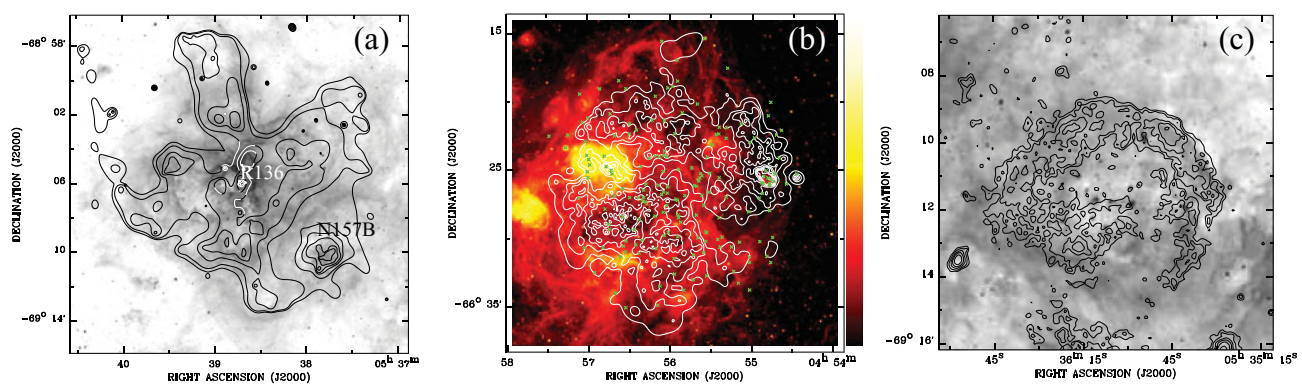

Figure 1. Examples of superbubbles in the LMC: (a) 30 Dor, (b) N11, and (c) 30 Dor C. Overlaid on the $\mathrm{H} \alpha$ images are X-ray intensity contours constructed in the Chandra ACIS-I 0.5-2 keV band for (a) and (b) and in the XMM-Newton MOS 0.4-1 keV band for (c). Discrete sources, as marked bycrosses, have been excised in (b). 

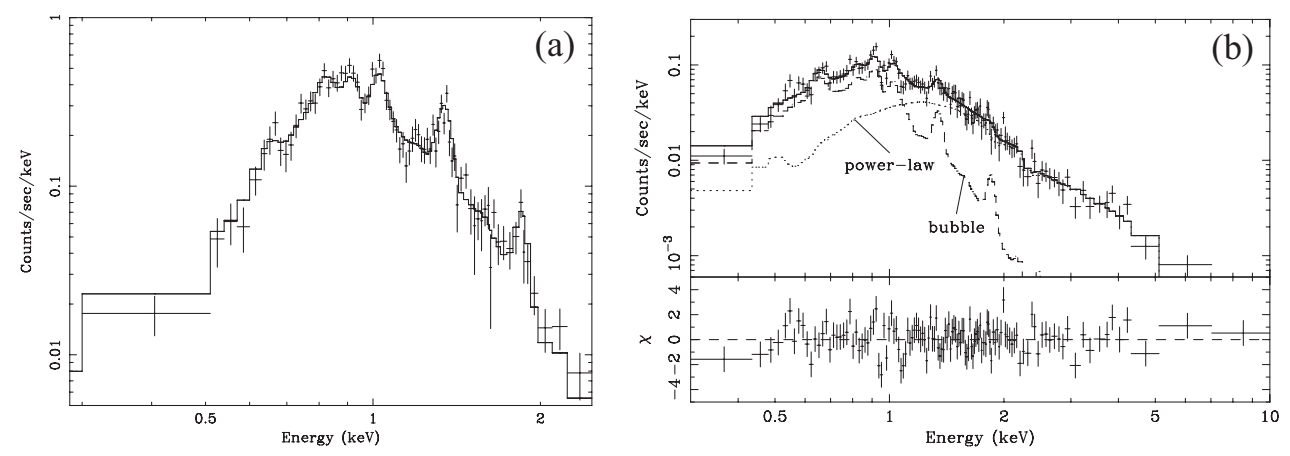

Figure 2. (a) Comparison of XMM-Newton MOS spectra of (a) 30 Dor, excluding R136 and SNR N157B (Fig. 1a) and (b) 30 Dor C.

Fleishman 1992). The energy efficiency of the CR-acceleration in SBs could reach up to about $30 \%$ at their early evolutionary stages (ages of a few $10^{6}$ years; e.g., Butt \& Bykov (2009)) Nonthermal X-ray emission has indeed been observed in a number of SBs, including 30 Dor C (Smith \& Wang 2004, Yamaguchi et al. (2010) in the LMC, IC131 in M33 (Tüllmann et al. 2009). Fig. 2 compares X-ray spectra of 30 Dor and 30 Dor C. While the former is dominated by thermal emission, the latter exhibits a substantially hard (apparently nonthermal) component (Smith \& Wang 2004).

All these processes still need to be carefully studied to determine their actual roles in regulating the energetics of SBs, which could have strong implications for our understanding the impact of massive stars on galaxy ecosystems.

\section{Hot gas in galactic spheroids}

Spheroids, including bulges of spiral galaxies and elliptical galaxies, are primarily comprised of old stars, which account for more than half of the stellar mass in the local Universe (Fukugita, Hogan, \& Peebles 1998). These stars collectively generate a substantial feedback in form of gradual stellar mass-loss and energetic Type Ia SNe. Understanding how this relatively gentle but long-lasting feedback affects galaxy evolution is one of the fundamental questions in astrophysics (e.g., Wang 2010). In order to address this issue, one needs an affective tracer of the ejected mass and energy in spheroids. While they typically contain little cool gas, one would expect that the mass should be mostly in hot gas, heated by the SNe. Indeed, diffuse optically-thin thermal X-ray emission has been observed in elliptical galaxies and in galactic bulges. However it has been shown repeatedly that the X-ray-inferred gas mass and energy are far less than those empirical predictions (e.g. David et al. 2006), which becomes particularly acute in X-ray-faint spheroids (bulges of spirals and low/intermediate-mass ellipticals). In such a spheroid, the luminosity of the X-ray emission accounts for no more than a few percent of the expected SNe energy input, and the deduced iron metallicity is typically sub-solar, inconsistent with the expected Ia SN enrichment. The inferred total mass of the X-ray-emitting hot gas represents only a small fraction of what is expected from the stellar mass loss over the galaxy's lifetime. These discrepancies clearly indicate that the stellar feedback in such a spheroid has gone with a wind or outflow (e.g., Bregman 1980; Ciotti et al. 1991; David et al. 2006; Tang et al. 2009a, 2009b; Tang \& Wang 2010).

The study of hot gas in galactic spheroids has quite a long history. Early works were based primarily on 1-D galactic wind modeling. Except for massive ellipticals, very hot $(>1 \mathrm{keV})$ and fast $(v>800 \mathrm{~km} / \mathrm{sec})$ spheroidal wind was predicted (e.g., Mathews \& Baker 1971; Ciotti et al. 1991). For such a hot wind, the inferred X-ray emission should 
be much less than $10^{36} \mathrm{ergs} \mathrm{s}^{-1}$ (e.g., Tang et al. 2009b), which is two orders of magnitude lower than the recently observed value of the galactic spheroid $\left(\sim 3 \times 10^{38} \mathrm{ergs} \mathrm{s}^{-1}\right.$ for the M31 bulge; Li \& Wang 2007; Bogdan \& Gilfanov 2008).

One important fact that the 1-D supersonic wind modeling does not account for is the discreteness of Ia SN heating. X-ray emission, proportional to the emission measure, is sensitive to the detailed structure of the hot gas. We have therefore conducted 3D simulations of SN-driven galactic winds (Tang et al. 2009b), in which the energy is assumed to come mainly from sporadic SNe and the mass mainly from stellar winds. The results are illustrated in Fig. 3. Our simulations confirm that Ia SNe produce a spheroidal wind, and more importantly, reveal its substructures. The bulk of the X-ray emission originates from the relatively low-temperature and low-abundance gas shells associated with SN blastwaves; the SN ejecta are not well mixed with the ambient medium. These results are qualitatively consistent with the apparent lack of evidence for iron enrichment in X-ray-faint galaxies. Compared to the 1-D wind model, the non-uniformity of simulated gas density, temperature, and metallicity in the 3-D simulations increases the diffuse X-ray luminosity by a factor of a few, narrowing the discrepancy between the theorypredicted and the observed X-ray emission. However, the resultant diffuse luminosities in the 3-D simulations is still more than one order of magnitude less than those observed in the Galactic and M31 bulges, indicating that gas in these spheroids is in a subsonic outflow state, probably due to additional mass loading to the hot gas and/or due to energy input rate that is substantially lower than the current estimate.

A subsonic outflow from a spheroid is intimately related to its formation and evolution history (Ciotti et al. (1991), Tang et al. (2009a)). We have developed a 1-D model of the spheroid outflow and galaxy accretion interplay. This model is based on 1-D hydrodynamic simulations and on an approximated dark matter accretion history of galaxies (Tang et al. 2009a). In our model, the outer boundary condition is the (supersonic) Hubble flow, which avoids the boundary problem. The spheroid wind/outflow occurs well inside the spatial range of the simulations. The feedback is assumed to consist of two primary phases: 1) an initial burst during the spheroid formation and 2) a subsequent long-lasting mass and energy injection from stellar winds and Ia SNe of evolved low-mass stars. These two phases of the feedback re-enforce each-other's impact on the gas dynamics. An outward blastwave is initiated by the burst and is maintained and enhanced by the long-lasting stellar feedback. For an M31-like bulge, for example, this blastwave can heat the surrounding medium not only in the galactic halo, but also in regions beyond the virial radius. The long-lasting feedback forms a galactic spheroid wind initially, which is reverse-shocked at a large radius, and may later evolve into a stable subsonic outflow as the energy injection gradually decreases with time. In a subsonic outflow, the properties of hot gas depend sensitively on the environment and formation history, which can explain the large dispersion of $L_{X} / L_{K}$ in early-type galaxies with similar $L_{K}$ as well as the missing stellar feedback problem (Wang 2010 and references therein). Therefore, the understanding of the interplay between the hot gas outflows and the large-scale galaxy environment is essential to the correct interpretation of observed diffuse X-ray emission in and around galactic spheroids.

Tang \& Wang (2010) have further conducted 3-D simulations based on the 1-D subsonic solution to explore combined effects. In addition to the expected enhanced X-ray emission, they find that SN reverse shock-heated iron ejecta is typically found to have a very high temperature and low density, hence producing little X-ray emission. Such hot ejecta, driven by its large buoyancy, can quickly reach a substantially higher outward velocity than the ambient medium, which is dominated by mass-loss from evolved stars. The 

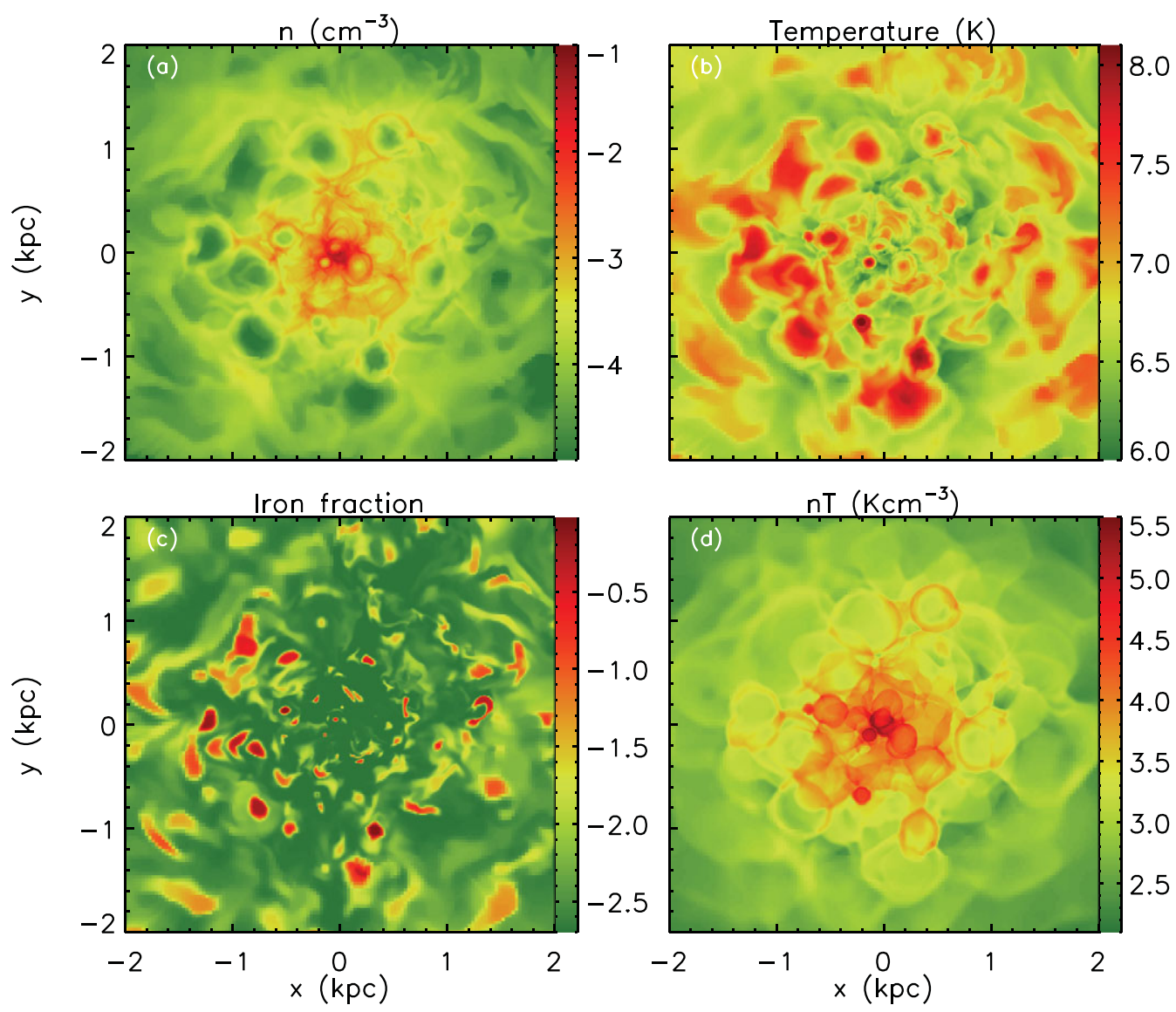

Figure 3. Density, temperature, iron mass fraction, and pressure of gas in a cut through a simulated spheroidal wind. The image values are scaled logarithmically. The upper-right octant has a higher resolution in the simulation, allowing for testing various resolution effects.

ejecta is gradually and dynamically mixed with the medium at large galactic radii. The ejecta is also slowly diluted and cooled by in situ mass injection from evolved stars. These processes together naturally result in the observed positive gradient in the average radial iron abundance distribution of the hot gas, even if mass weighted. This trend is in addition to the X-ray measurement bias that tends to underestimate the iron abundance for the hot gas with a temperature distribution.

One limitation of our previous simulations is that the mass loss from evolved stars takes only the form of continuous input, following the average stellar distribution of a galactic spheroid. In reality, however, the continuous wind, mainly from constant mass loss of numerous giant stars, contributes only about half of the stellar mass loss in the spheroid (e.g. Buckley \& Schneider 1995). The other half of mass loss occurs in the planetary nebula $(\mathrm{PN})$ stage of intermediate and low mass stars. This mass loss is an approximately impulsive mass-loss event at the end of the life of such a star and lasts only $\sim 10^{3}$ years. The ejecta forms an expanding $(\sim 10 \mathrm{~km} / \mathrm{s})$ and cold $\left(\lesssim 10^{5} \mathrm{~K}\right)$ gas shell. Naturally, one wants to know whether or not the PN ejecta can be heated by shocks and/or by thermal mixing with the hot ambient gas, or how the discreteness of the mass input can affect the diffuse X-ray emission of a spheroid. 

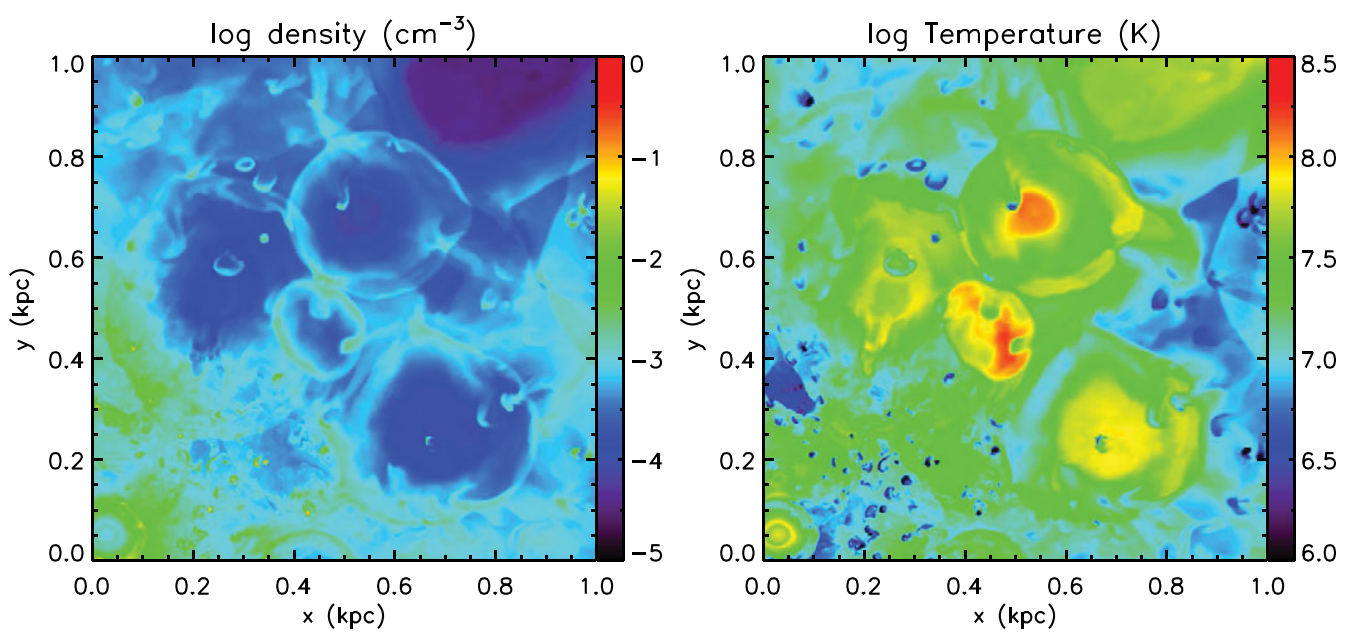

Figure 4. A snapshot of our pilot simulation, including discrete PNe. Presented here are density (left) and temperature (right), viewed in a cut through the central $1 \mathrm{kpc} \times 1 \mathrm{kpc}$ region of a spheroid. The simulation, which includes all three types of feedback $(\mathrm{SNe}+$ stellar winds + $\mathrm{PNe}$ ), has evolved 1.3 Myr after discrete PN seeds start to be planted.

Bregman \& Parroit (2009) have conducted 2-D hydrodynamic simulations of an individual PN undergoing ram-pressure stripping of ambient hot gas in a typical spheroid environment. They show that fluid instabilities result in mixing and heating of about half of the PN ejecta to $10^{5}-10^{6} \mathrm{~K}$, a temperature range that is still at least a factor of several lower than that of the hot gas, while the other half remains cold $\left(<10^{5} \mathrm{~K}\right)$ and flows out of the simulation box ( $25 \mathrm{pc}$ long in the opposite direction of the motion). These results provide useful insights into the PNe feedback processes. But clearly the simulations have several major limitations, in terms of the explored volume, strength/mode of fluid instabilities, and interplay among various objects/processes in a spheroid.

In a real galactic spheroid, the environment can be very violent because of the presence of SN shockwaves and the collisions among randomly moving PNe, as well as the global spheroid-wide outflow, and hence the large pressure and density gradients. We have recently performed a pilot study, which for the first time simulates a galactic spheroid with discrete PN feedback. While the basic setting is the same as that described in Tang \& Wang (2010), we make the PN mass contribution discrete, after the SN-driven galactic outflow becomes stable (Tang \& Wang 2010). The positions and velocities of the imbedded PN seeds, with an occurrence rate of $4.5 \times 10^{4} \mathrm{Myr}^{-1}$, are determined in a separate N-body simulation using the software package Zeno (developed by Joshua Barnes). Each embedded PN seed has a mass of $0.3 \mathrm{M}_{\odot}$, an expansion velocity of $20 \mathrm{~km} \mathrm{~s}^{-1}$, and a uniform temperature of $10^{5} \mathrm{~K}$ (artificially set to achieve a sufficiently high initial resolution). The size of the seed is determined by a rough local pressure balance at the embedded position. The refinement level reaches down to $\sim 1 \mathrm{pc}$. The embedded PNe then continue to expand and interact with their environments.

Fig. 4 illustrates the result of the simulation, showing a diversity of PN morphologies on scales of a few pc to a few tens of pc, while SN shock waves are typically on larger scales. Close examination of individual PNe suggests that some of them are morphologically similar to that in the 2-D simulations (Bregman \& Parroit 2009), but others appear to be very different, demonstrating complicated interaction of $\mathrm{PNe}$ with the environments. In the inner most region where the gas density and stellar number density are high, PNe only grow to several pc in size, while in outer regions or inside low-density SNRs 
PNe can grow as big as 50 pc. Most interestingly, many of the PNe seem to be heated eventually to a couple of million degrees, at which the bulk of the observed diffuse X-ray luminosity is emitted. Therefore, the debris of PNe can naturally lead to enhanced X-ray emission. Detailed simulation and analysis will enable us to examine the evolution of individual PNe to see how instabilities grow in different local environments, to quantify what fraction of PN mass is heated to a temperature high enough to emit X-ray, and to check whether or not the enhanced X-ray emission could make up the difference between previous simulation predicted value and the observed one.

In conclusion, most SNRs are not observed individually. These "missing" SNRs collectively represent a key component of the stellar feedback in galaxies, in either active star-forming spirals or cool gas-poor spheroids. The feedback regulates the ecosystems of the galaxies, hence their evolution.

\section{References}

Bogdan A. \& Gilfanov M. 2008, MNRAS, 388, 56

Bregman, J. N. 1980, ApJ, 237, 280

Bregman, J. N \& Parriott, J. R. 2009, ApJ, 699, 923

Buckley, D. \& Schneider, S. E. 1995, ApJ, 446, 279

Butt, Yousaf M., \& Bykov, Andrei M. 2008, ApJ (Letters) 677, 21

Bykov, A. M. \& Fleishman, D. G. 1992, MNRAS, 255, 269

Cho, H. \& Kang, H., 2008, New Astron., 13, 163

Chu, Y.-H. \& Mac Low, M. 1990, ApJ, 365, 510

Ciotti, L., Pellegrini, S., Renzini, A., \& D'Erocole, A. 1991, ApJ, 376, 380

David L. P., Jones C., Forman W., Vargas I. M., \& Nulsen P., 2006, ApJ, 653, 207

Dong, H., Wang, Q. D., \& Morris, M. R. 2012, MNRAS, 425, 884

Everett, J. E. \& Churchwell, E. 2010, ApJ, 713, 592

Fisk, L. A. \& Gloeckler, G. 2012, ApJ, 744, 127

Fukugita M., et al. 1998, ApJ, 503, 518

Higdon, J. C. \& Ligenfelter, R. E. 2005, ApJ, 628, 738

Jaskot, A. E. et al. 2011, ApJ, 729, 28

Krause, M. et al. 2013, A\&\&A, 550, 49

Li. Z. Y., \& Wang, Q. D. 2007, ApJ, 668, L39

Mac Low, M.-M. \& McCray, R. 1988, ApJ, 324, 776

Mathews, W. G. \& Baker, J. C. 1971, ApJ, 170, 241

Monaco, P. 2004, MNRAS, 354, 151

Oey, M. S. 2009, in AIP Conf. Ser. 1156, The Local Bubble and Beyond II, ed.: R. K. Smith,

S. L. Snowden, \& K. D. Kuntz (Melville, NY: AIP), 295

Parizot, E., et al. 2004, A\& $A, 424,747$

Rajagopal, J. et al. 2007, ApJ, 671, 2017

Smith, D. A. \& Wang, Q. D. 2004, ApJ, 611, 881

Tüllmann, R., et al. 2009, ApJ, 707, 1361

Tang, S. K. \& Wang, Q. D. 2005, ApJ, 628, 205

Tang, S. K. \& Wang, Q. D. 2009, 397, 2106

Tang, S. K., Wang, Q. D., Lu, Y., \& Mo, H. J. 2009a, MNRAS, 392, 77

Tang, S. K., Wang, Q. D., Joung, M. K. R., \& Mac Low, M. M. 2009b, 398, 1468

Tang, S. K. \& Wang, Q. D. 2010, MNRAS, 408, 1011

Wang, Q. \& Helfand, D. J. 1991, ApJ, 373, 497

Wang, Q. D. 2010, PNAS, 107, 7168

Weaver, et al. 1977, ApJ, 218, 377

Yamaguchi, H., Sawada, M., \& Bamba, A. 2010, ApJ, 715, 412 


\section{Discussion}

Podsiadlowski: What was the assumed SN Ia note in your MB1 simulation? I guess for a X-ray efficiency of $\sim 1 \%$ you need about 1 SN every $10^{3} \mathrm{yr}$ ?

WANG: The standard SN Ia rate as a fraction of stellar mass is used. 